\title{
Análisis biogeográfico de la diversidad pteridofítica en Argentina y Chile continental
}

\author{
Biogeographical analysis of the pteridophyte diversity of continental \\ Argentina and Chile
}

MÓNICA PONCE ${ }^{1}$, KLAUS MEHLTRETER $^{2} \&$ ELÍAS R. DE LA SOTA ${ }^{3}$

\begin{abstract}
${ }^{1}$ Instituto de Botánica Darwinion, Academia Nacional de Ciencias Exactas, Físicas y Naturales y
CONICET, Casilla de Correo 22, 1642 San Isidro, Argentina; e-mail: mponce@ darwin.edu.ar ${ }^{2}$ Instituto de Ecología, Departamento de Ecología Vegetal, Apartado Postal 63, 91000 Xalapa, Veracruz, México; e-mail: klaus@ecologia.edu.mx

${ }^{3}$ Facultad de Ciencias Naturales y Museo, Universidad Nacional de La Plata, Paseo del Bosque s/n, 1900

La Plata, Argentina; e-mail: sota@museo.fcnym.unlp.edu.ar
\end{abstract}

\begin{abstract}
RESUMEN
Las Pteridófitas de Chile continental están representadas por 116 especies nativas (43 géneros, 20 familias), y en Argentina continental por 346 (86 géneros, 27 familias). Ambos países comparten 89 especies (41 géneros, 20 familias). La menor diversidad pteridofítica y el mayor endemismo en Chile $(11,2 \%)$ que en Argentina $(3,5 \%)$ están relacionados probablemente con extinciones históricas y su actual aislamiento geoclimático. Sin embargo, se destaca que el endemismo en los helechos es cinco a seis veces menor que en Angiospermas. Los géneros con más especies en Argentina son Thelypteris (34), Asplenium (32), Cheilanthes (21), Blechnum (19) e Hymenophyllum (16). Los dos últimos también son los más diversificados en Chile (Hymenophyllum, 18 y Blechnum, 10). En la región estudiada la diversidad muestra incrementos latitudinales opuestos, concentrándose en tres centros, dos subtropicales en el noroeste y noreste de Argentina y uno templado-lluvioso en el sur argentino-chileno alrededor de los $40^{\circ}$ latitud sur. Los tres centros de diversidad concentran el $93 \%$ de las especies y el $95 \%$ de los endemismos, compartiendo pocas especies entre ellos. En los centros subtropicales las familias Aspleniaceae, Polypodiaceae, Pteridaceae, Thelypteridaceae y Selaginellaceae son sus componentes más importantes, y comparten un gran número de especies con Bolivia y Brasil, respectivamente. El centro templado sur tiene una menor diversidad pteridofítica, pero se hallan en él mayor cantidad de endemismos (77\%) que en los centros subtropicales, a consecuencia de un fuerte aislamiento. Sin embargo, presenta altos índices de similitud e intercambio florístico entre ambas vertientes de Los Andes. Allí las Blechnaceae y Hymenophyllaceae son las familias mejor representadas. Los taxa que presentan una distribución disyunta encontrándose en los tres centros de diversidad, nos indican que la pteridoflora del Cono Sur fue históricamente más extensa y continua, y que sus límites han retrocedido a causa de los impactantes cambios climáticos y geomorfológicos que sucedieron durante el Terciario y el Pleistoceno.
\end{abstract}

Palabras clave: Pteridófitas, biogeografía, diversidad, Argentina, Chile.

\begin{abstract}
The pteridophytes are represented by 116 native species ( 43 genera/20 families) in continental Chile, and by 346 native species (86 genera/27 families) in continental Argentina. Both countries share 89 species (41 genera/20 families). Lower pteridophyte diversity and the higher endemism in Chile (11.2\%) than in Argentina (3.5\%) are possibly related to historical extinctions and the actual geo-climatic isolation. However, the endemism in pteridophytes is five to six times lower than in angiosperms. The richest Argentinean genera are Thelypteris (34 species), Asplenium (32), Cheilanthes (21), Blechnum (19) and Hymenophyllum (16). The last two genera are also the most numerous in Chile (Hymenophyllum 18 species and Blechnum 10). In the two countries, pteridophyte diversity shows opposite latitudinal trends, with high species richness in three centers: two subtropical humid centers in northwestern and northeastern Argentina, and a temperate humid center in southern Chile and Argentina around $40^{\circ} \mathrm{S}$. The three centers concentrate $93 \%$ of the species and $95 \%$ of the endemisms, sharing few species. In the subtropical centers, the families Aspleniaceae, Polypodiaceae, Pteridaceae, Thelypteridaceae and Selaginellaceae are the main components and share a large number of species with Bolivia and Brazil, respectively. The southern temperate center has a lower pteridophyte diversity, but a larger number of endemisms (77\%) than the northern subtropical centers. However, adjacent regions of Chile and Argentina within the temperate center have high similarity and maintain a strong floristic interchange. Blechnaceae and Hymenophyllaceae are the most common families. Taxa with disjunct distribution patterns and with presence in the three centers indicate that the pteridophyte flora had a wider and continuous distribution in the past, and that their limits have receded as a consequence of the strong climatic and geomorphologic changes during the Tertiary and the Pleistocene.
\end{abstract}

Key words: Pteridophytes, biogeography, diversity, Argentina, Chile. 


\section{INTRODUCCIÓN}

Las Pteridófitas presentan características propias de dispersión y distribución, en comparación con las Angiospermas (Tryon 1986, Barrington 1993). La producción de esporas es inmensa (100.00030.000 millones por individuo), las esporas tienen menor tamaño que las semillas $(30-50 \mu \mathrm{m})$, son dispersadas por el viento y su distribución geográfica es más amplia (Kramer et al. 1995). Además, por su menor número de taxa y la existencia de numerosos trabajos sobre su sistemática en Sudamérica austral (ver Ponce 1996), cumplen con el requisito de ser un grupo taxonómico bien conocido (Kato 1993, Smith 1993), apropiado para un estudio biogeográfico.

Varios autores han analizados la distribución de las Pteridófitas de Chile (Looser 1932, Godoy \& Figueroa, 1989; Rodríguez 1989, 1995a) y de Argentina (Hicken 1919), siendo de la Sota (1973a) el primero en considerar todo el Cono Sur. Sin embargo, no existen estudios comparativos de ambas regiones. El objetivo del presente artículo es: (1) realizar un primer análisis cuantitativo que abarca la flora pteridofítica de ambos países en base al conocimiento actualizado de su sistemática para precisar la distribución a nivel de especies, géneros y familias; (2) identificar los centros de diversidad y sus endemismos; (3) identificar las áreas de intercambio florístico más intenso entre los centros de diversidad y sus áreas adyacentes; (4) discutir las posibles causas climáticas, geomorfológicas y ecológicas para los patrones observados.

La región austral de América es un territorio con características climáticas marcadamente oceánicas, atravesado por la Diagonal Árida que abarca el norte de Chile y el sureste de Argentina (Fig. 1). La Diagonal Árida y la Cordillera de Los Andes son las barreras climáticas y geomorfológicas más evidentes del Cono Sur. Mientras la primera barrera limita el intercambio florístico entre el noreste y el suroeste del Cono Sur, la segunda complica el intercambio entre el este y el oeste. Entre el norte del Cono Sur y las áreas adyacentes tropicales de Bolivia y Brasil no existen barreras considerables. Históricamente la glaciación del Pleistoceno (Villagrán et al. 1996) fue el evento más importante que afectó el Cono Sur de América, especialmente a partir de los $45^{\circ}$ de latitud sur. ¿Cómo coinciden los actuales patrones de distribución de las Pteridófitas con estos factores climáticos, geomorfológicos e históricos mencionados?

\section{MATERIALES Y MÉTODOS}

La información publicada sobre las floras pteridofíticas de Argentina y Chile (Looser 1948, 1955, 1961, 1962, 1966, 1968, Duek \& Rodríguez 1972, de la Sota 1972, 1973b, 1976, 1977a, 1977b, Ponce 1994, 1995, 1996, Rodríguez 1995b, de la Sota et al. 1998) se ingresó en la base de datos FPL (Flora Pteridológica Latinoamericana), una aplicación construida en el programa MS-Access ${ }^{\circledR}$ (Mehltreter \& Palacios-Rios 1998', Palacios-Rios \& Mehltreter 1998). La taxonomía se basó en el sistema de clasificación de Kramer \& Green (1990) a nivel de familias, y de Rodríguez (1995 b) y Ponce (1996) a nivel de géneros. Se excluyeron del análisis los taxa introducidos o exóticos subespontáneos (tres especies en Chile (Adiantum capillus-veneris, Dryopteris filix-mas, Selaginella apoda) y 10 especies en Argentina (Ceterach officinarum, Cyrtomium falcatum, Dryopteris filix-mas, Macrothelypteris torresiana, Nephrolepis cordifolia, Pteris cretica, Pteris multifida, Pteris tremula, Pteris vittata, Thelypteris dentata). Además se excluyeron los taxa considerados como de «presencia probable» en la región estudiada, y los taxa subespecíficos. Sin embargo, los últimos fueron considerados para determinar los endemismos (Anexo 1).

Se determinó la distribución y el endemismo de cada familia, género y especie en cada una de las 13 regiones políticas de Chile y 23 provincias de Argentina. Se establecieron las áreas de distinta concentración de especies, graficándolas en el mapa como isolíneas. La confección del mismo se apoyó además en datos propios y en la información de mapas de vegetación, clima y fisiográficos (Cabrera \& Willink 1973, Argentina-IGM 1998, Chile-IGM 1985).

Para comparar la riqueza de especies en áreas de diferentes tamaños, se aplicó el índice de biodiversidad taxonómica (Squeo et al. 1998), que es el número de especies dividido por el logaritmo natural del área en kilómetros cuadrados. Para determinar la similitud florística entre los centros de diversidad se usaron los índices de Jaccard $\left(\mathrm{C} /\left(\mathrm{N}_{1}+\mathrm{N}_{2}-\mathrm{C}\right)\right)$ y Simpson $\left(\mathrm{C} / \mathrm{N}_{1}\right)$, donde $\mathrm{C}$ es el número de especies compartidas, $\mathrm{N}_{1}$ el

\section{${ }^{1}$ MEHLTRETER K \& M PALACIOS-RÍOS} (1998) Flora pteridológica latinoamericana: una base de datos florística y biogeográfica para los helechos y plantas afines de Latinoamérica. Séptimo Congreso Latinoamericano de BotánicaDécimo Cuarto Congreso Mexicano de Botánica, México, Distrito Federal, Resumen: 230. 


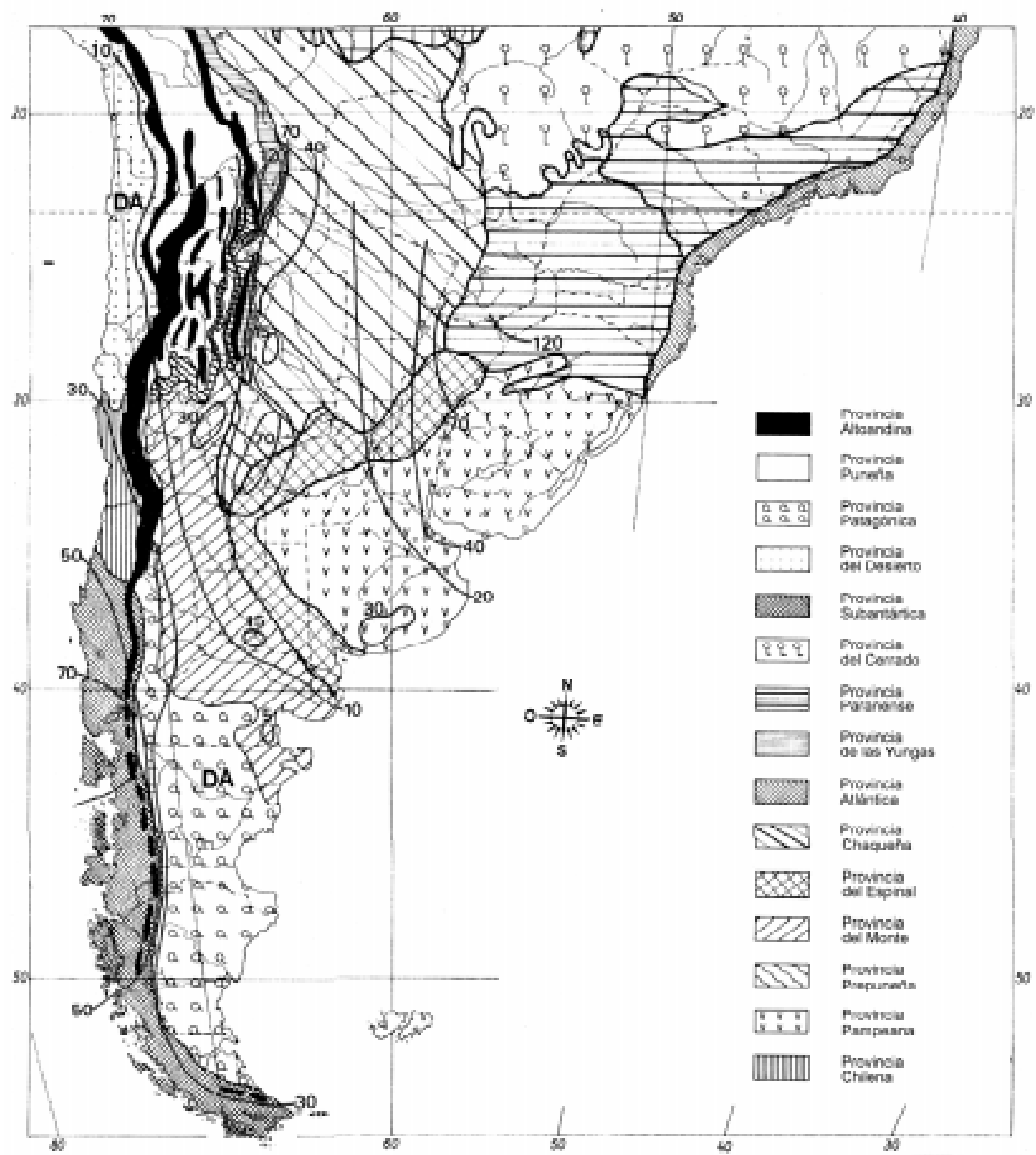

Fig. 1: Provincias biogeográficas de Cabrera \& Willink (1973), Diagonal Árida de Sudamérica (DA) e Isolíneas de números de especies.

Biogeographic provinces of Cabrera \& Willink (1973), Arid Diagonal of South America (DA) and Isolines of species numbers.

número de especies en el área de menor diversidad y $\mathrm{N}_{2}$ el número de especies en la segunda área de mayor diversidad (Brown \& Lomolino 1998). Se prefirió el índice de Simpson para comparaciones con regiones donde no se conoce el número total de especies, como en el caso de Bolivia, Brasil y Paraguay. El índice de Jaccard varía entre 0 y 1 y es equivalente al porcentaje de especies compartidas. Para comparar estadísticamente la similitud florística entre los dos países a nivel de familias y géneros, se utilizó el coeficiente de correlación de Spearman $\mathrm{r}_{\mathrm{s}}$ (Zar 1999).

\section{RESULTADOS}

El número de taxa nativos de Chile y de Argentina y el número de taxa compartidos entre ambos países, se muestra en la Tabla 1. Las 7 familias 
ausentes en Chile continental son: Cyatheaceae, Dicksoniaceae, Marattiaceae, Osmundaceae, Psilotaceae, Selaginellaceae y Vittariaceae. En promedio el número de especies por familia en el Cono Sur es tres veces mayor en Argentina que en Chile, como lo indica la pendiente de la regresión (Chile $=0,42+0,30 \times$ Arg). Esta relación indica que familias con más especies en Argentina son también las más abundantes en Chile y viceversa (Fig. 2), con excepción de las Polypodiaceae y Thelypteridaceae, que tienen una muy baja diversidad en Chile. Así como en las familias muchos géneros son más diversos en Argentina, con un promedio de 4,0 especies por género y en Chile 2,7 (Tabla 2). Los géneros más diversificados de Chile continental son Hymenophyllum (18) y Blechnum (10), mientras en Argentina éstos son Thelypteris (34 especies) y Asplenium (32). El género Trichomanes es el único de los 41 géneros compartidos que no tiene ninguna especie en común. Los 40 géneros restantes comparten 89 especies comunes, de cuales se destacan Lycopodium, Hymenophyllum y Polystichum con los mayores índices de Jaccard de los géneros con más de cinco especies (Tabla 1 y 2).

De acuerdo al índice de Squeo et al. (1998), que considera el área en una escala logarítmica, Argentina $(23,3)$ tendría una mayor diversidad taxonómica que Chile $(8,6)$.

\section{Centros de diversidad}

Se determinaron tres centros de diversidad, dos centros subtropicales en el noroeste (CSNO) y nordeste (CSNE) de Argentina, y un tercero en la región templada sur (CTS) de ambos países alrededor de los $40^{\circ}$ latitud (Fig. 3 y Tabla 3). El CSNE abarca la provincia de Misiones, el CSNO incluye Jujuy, Salta y Tucumán,y el CTS se ubica en los Andes templados y templado-fríos, comprendiendo las regiones de Los Lagos, Biobio, Araucanía y Aisén y las provincias de Río Negro y Neuquén.

Estos tres centros concentran el $93 \%$ de todas las especies (344 especies). Los dos centros

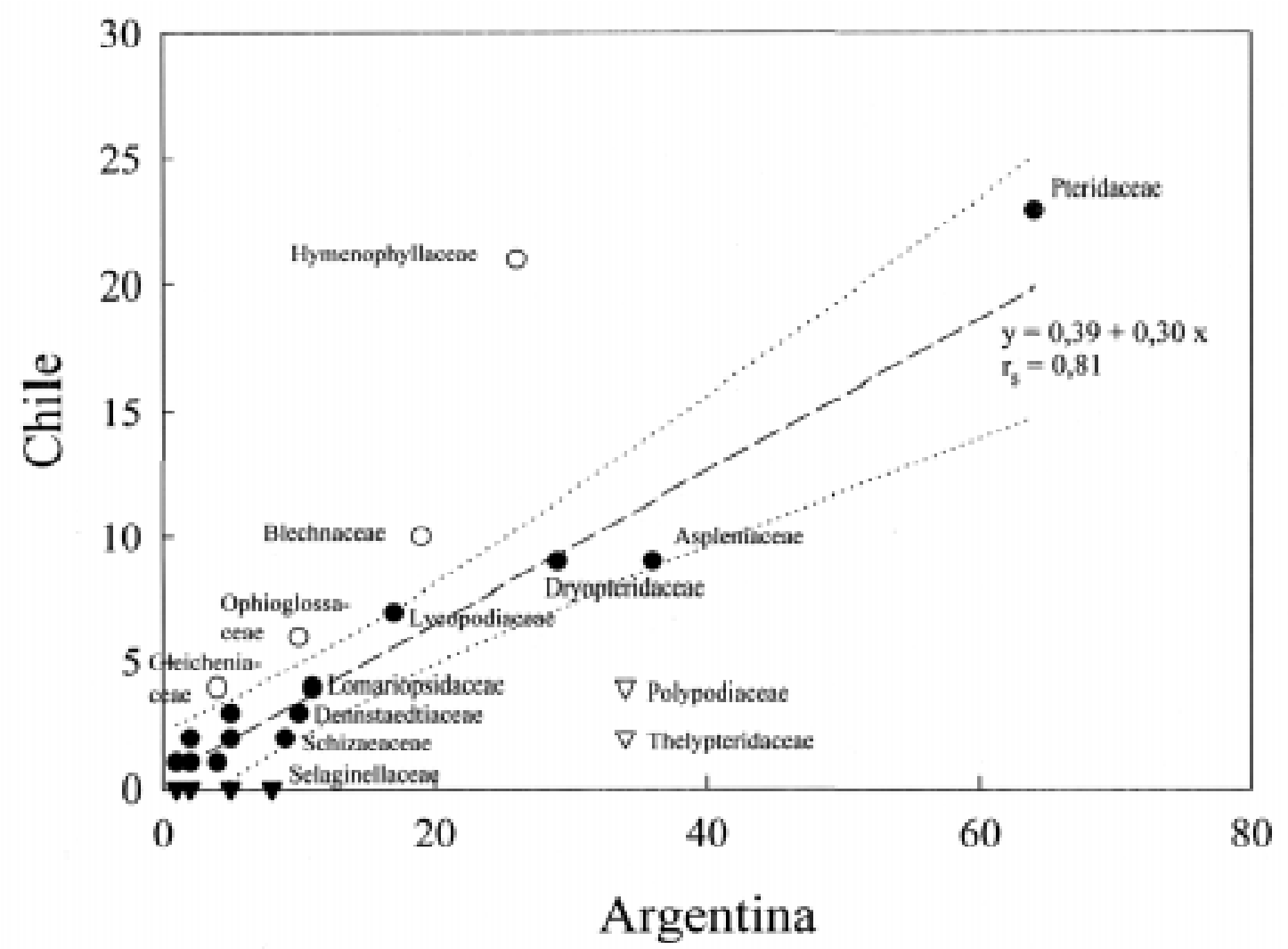

Fig. 2: Número de especies para las 27 familias de Pteridófitas en Argentina y Chile. Familias suprarepresentadas (círculos vacíos), igualmente representadas (círculos rellenos), sub-representadas (triángulos vacíos), y no representadas (triángulos rellenos) en Chile.

Species number of the 27 Pteridophyte families of Argentina and Chile. For Chile, overrepresented (empty circles), equally represented (full circles), subrepresented (empty triangles), and not represented (full triangles) families are indicated. 
TABLA 1

Número de taxa nativos de Pteridófitas en las floras de Chile y Argentina continentales. Los taxa exclusivos ("excl") son los que sin ser endémicos no se encuentran en ambos países. Se excluyeron las 10 especies introducidas en la región

Number of native, exclusive and shared taxa (the abbreviation "excl" refers to non-endemic taxa, absent in one of both countries). The 10 introduced species of the region were excluded

\begin{tabular}{lcccccc}
\hline $\begin{array}{l}\text { Nivel } \\
\text { taxonómico }\end{array}$ & \multicolumn{2}{c}{ Argentina } & \multicolumn{2}{c}{ Chile } & Taxa (\%) & Total \\
Total & Excl & Total & Excl & compartidos & \\
\hline Familias & 27 & 7 & 20 & 0 & $20(71,4)$ & 27 \\
Géneros & 86 & 45 & 43 & 2 & $41(47,7)$ & 88 \\
Especies & 346 & 257 & 116 & 27 & $89(24,3)$ & 373 \\
\hline
\end{tabular}

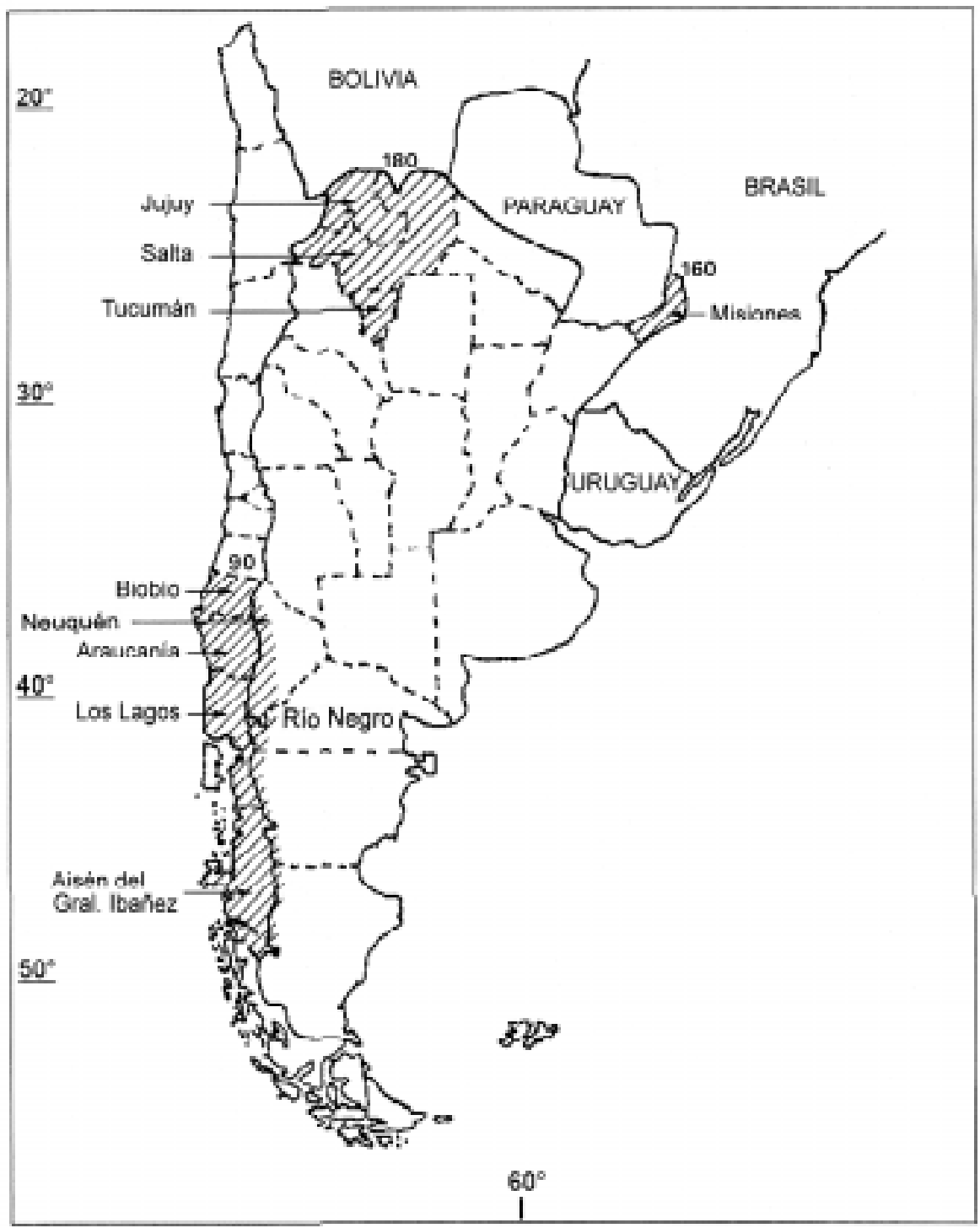

Fig. 3: Centros de diversidad pteridofítica: Centro Subtropical del NE (CSNE) de Argentina, Centro Subtropical del NO (CSNO) de Argentina y Centro Templado del S (CTS) de Chile y Argentina. Centers of pteridophyte diversity: Subtropical Center of Northeastern Argentina (CSNE), Subtropical Center of Northwestern Argentina (CSNO) and Temperate Center of Southern Chile and Argentina (CTS). 
subtropicales reúnen el $74 \%$ (276 especies) con un predominio de Aspleniaceae, Polypodiaceae, Pteridaceae y Thelypteridaceae. El centro templado es caracterizado por Blechnaceae, Grammitidaceae, Lycopodiaceae e Hymenophyllaceae. Valores similares en los tres centros muestran las Dryopteridaceae (Fig. 4).

Al analizar la diversidad (Squeo et al. 1998) para los tres centros (Tabla 3), se observa que la diversidad $(15,6)$ en el CSNE es similar a la del CSNO $(14,7)$, a pesar de que Misiones es la octava parte del área comprendida por las 3 provincias del noroeste. La riqueza específica $(7,0)$ en la región sur se reduce a la mitad teniendo en cuenta la enorme diferencia de área y en variedad de ambientes, algunos no favorables para los helechos. Sin embargo es una diversidad apreciable si se la compara con los índices de provincias con

TABLA 2

Comparación de los 41 géneros compartidos entre Argentina y Chile: número de especies para cada género y país, y número de especies compartidas, ordenada por el Índice de Jaccard

Comparison of the 41 shared genera between Argentina and Chile, species number per genus and country and number of common species, sorted by the Jaccard' Index

\begin{tabular}{|c|c|c|c|c|c|}
\hline Género & Familia & Argentina & Chile & $\begin{array}{c}\text { Especies } \\
\text { compartidas }\end{array}$ & $\begin{array}{c}\text { Índice de } \\
\text { Jaccard }\end{array}$ \\
\hline Trichomanes & Hymenophyllaceae & 9 & 1 & 0 & 0,00 \\
\hline Thelypteris & Thelypteridaceae & 34 & 2 & 2 & 0,06 \\
\hline Polypodium & Polypodiaceae & 10 & 3 & 1 & 0,08 \\
\hline Adiantum & Pteridaceae & 13 & 6 & 2 & 0,12 \\
\hline Huperzia & Lycopodiaceae & 7 & 1 & 1 & 0,14 \\
\hline Pteris & Pteridaceae & 6 & 2 & 1 & 0,14 \\
\hline Anemia & Schizaeaceae & 6 & 1 & 1 & 0,17 \\
\hline Asplenium & Aspleniaceae & 32 & 8 & 6 & 0,18 \\
\hline Elaphoglossum & Lomariopsidaceae & 9 & 4 & 2 & 0,18 \\
\hline Megalastrum & Dryopteridaceae & 5 & 1 & 1 & 0,20 \\
\hline Pellaea & Pteridaceae & 3 & 2 & 1 & 0,25 \\
\hline Salvinia & Salviniaceae & 4 & 1 & 1 & 0,25 \\
\hline Blechnum & Blechnaceae & 19 & 10 & 7 & 0,32 \\
\hline Cheilanthes & Pteridaceae & 21 & 8 & 7 & 0,32 \\
\hline Dennstaedtia & Dennstaedtiaceae & 3 & 1 & 1 & 0,33 \\
\hline Marsilea & Marsileaceae & 3 & 1 & 1 & 0,33 \\
\hline Isoëtes & Isoetaceae & 5 & 2 & 2 & 0,40 \\
\hline Ophioglossum & Ophioglossaceae & 6 & 4 & 3 & 0,43 \\
\hline Azolla & Azollaceae & 2 & 1 & 1 & 0,50 \\
\hline Cystopteris & Dryopteridaceae & 2 & 1 & 1 & 0,50 \\
\hline Gleichenia & Gleicheniaceae & 2 & 4 & 2 & 0,50 \\
\hline Hypolepis & Dennstaedtiaceae & 2 & 1 & 1 & 0,50 \\
\hline Pleopeltis & Polypodiaceae & 2 & 1 & 1 & 0,50 \\
\hline Polystichum & Dryopteridaceae & 6 & 5 & 4 & 0,57 \\
\hline Botrychium & Ophioglossaceae & 3 & 2 & 2 & 0,67 \\
\hline Hymenophyllum & Hymenophyllaceae & 16 & 18 & 14 & 0,70 \\
\hline Lycopodium & Lycopodiaceae & 8 & 6 & 6 & 0,75 \\
\hline Argyrochosma & Pteridaceae & 1 & 1 & 1 & 1,00 \\
\hline Astrolepis & Pteridaceae & 1 & 1 & 1 & 1,00 \\
\hline Cryptogramma & Pteridaceae & 1 & 1 & 1 & 1,00 \\
\hline Equisetum & Equisetaceae & 2 & 2 & 2 & 1,00 \\
\hline Grammitis & Grammitidaceae & 3 & 3 & 3 & 1,00 \\
\hline Lophosoria & Lophosoriaceae & 1 & 1 & 1 & 1,00 \\
\hline Notholaena & Pteridaceae & 1 & 1 & 1 & 1,00 \\
\hline Pilularia & Marsileaceae & 1 & 1 & 1 & 1,00 \\
\hline Pleurosorus & Aspleniaceae & 1 & 1 & 1 & 1,00 \\
\hline Rumohra & Dryopteridaceae & 1 & 1 & 1 & 1,00 \\
\hline Schizaea & Schizaeaceae & 1 & 1 & 1 & 1,00 \\
\hline Serpyllopsis & Hymenophyllaceae & 1 & 1 & 1 & 1,00 \\
\hline Trismeria & Pteridaceae & 1 & 1 & 1 & 1,00 \\
\hline Woodsia & Dryopteridaceae & 1 & 1 & 1 & 1,00 \\
\hline
\end{tabular}


Clima, familias más representadas, número de especies, endemismos y riqueza pteridofítica en tres centros de diversidad, similitud entre ellos, expresado como número de especies compartidas y por el índice de Jaccard. Abreviaturas: CSNE, Centro Subtropical del Nordeste de Argentina; CSNO, Centro Subtropical del Noroeste de Argentina; CTS, Centro Templado Sur de ambos países

Climate, best represented families, species number, number of endemisms and pteridophyte diversity in the three centers, and similarity between them, expressed as number of common species and as Jaccard Index. Abbreviations: CSNE, Subtropical Center of Northeastern Argentina; CSNO, Subtropical Center of Northwestern Argentina; CTS, Temperate Center of Southern Chile and Argentina

\begin{tabular}{|c|c|c|c|c|c|c|}
\hline Centros & Clima & $\begin{array}{l}\text { Familias más } \\
\text { representadas }\end{array}$ & $\begin{array}{c}\text { Número de } \\
\text { especies }\end{array}$ & $\begin{array}{l}\text { Número de } \\
\text { endemismos }\end{array}$ & $\begin{array}{c}\text { Especies } \\
\text { compartidas con } \\
\text { otros centros }\end{array}$ & $\begin{array}{c}\text { Índice de } \\
\text { Jaccard }\end{array}$ \\
\hline CSNE & Subtropical-húmedo & $\begin{array}{c}\text { Pteridaceae } \\
\text { Polyopodiaceae }\end{array}$ & 161 & 0 & 68 con $\mathrm{CSNO}$ & 0,25 \\
\hline CSNO & Subtropical-húmedo & $\begin{array}{c}\text { Pteridaceae } \\
\text { Thelypteridaceae }\end{array}$ & 182 & 10 & 21 con $\mathrm{CTS}$ & 0,08 \\
\hline CTS & Templado-húmedo & $\begin{array}{c}\text { Hymenophyllaceae } \\
\text { Blechnaceae }\end{array}$ & 92 & 44 & 7 con $\mathrm{CSNE}$ & 0,03 \\
\hline
\end{tabular}

menor superficie, como Maule $(3,0)$ y Valparaíso $(3,2)$ de Chile central y con provincias extensas y con climas aparentemente más propicios para las Pteridófitas como Corrientes $(6,0)$ y Entre Ríos $(3,3)$ en la Mesopotamia argentina.

La Fig. 1 muestra las isolíneas de diversidad. Representan aproximaciones de los números de especies por región, orientándose sobre la base de zonas de vegetación, altitud y precipitación. Las isolíneas no se completaron donde no se contaba con datos suficientes y en los límites del área de estudio. Además de los tres centros de diversidad, existen zonas que llaman la atención por su concentración pteridofítica dentro de la Diagonal Árida y la planicie chaco-pampeana. Son macizos montañosos más o menos aislados, como las Sierras de Guasayán, en Santiago del Estero, las de Famatina, Velasco, Valle Fértil, La Huerta, en La Rioja y San Juan, las de Córdoba y San Luis, australes de Buenos Aires, las de Lihuel Calel y Mahuidas, en La Pampa y la Meseta de Somuncurá y Sierra Grande, en Río Negro.

En Chile central habría áreas discontinuas de concentración de Pteridófitas en los bosques aislados ubicados a lo largo de la costa desde los $30^{\circ}$ $38^{\circ}$ de latitud sur (Villagrán \& Armesto 1980).

\section{Intercambio florístico}

Los tres centros de diversidad tienen relativamente pocas especies en común, como lo demuestran bajos índices de Jaccard. Mientras los dos centros subtropicales comparten una de cada cuatro especies (Índice de Jaccard $=0,25$ ), cada uno de estos centros tiene una baja similitud florística con el CTS (Tabla 3). Es en este CTS, donde los territorios adyacentes de Argentina y Chile tienen la mayor similitud florística (Tabla 4). Los dos centros subtropicales comparten muchas especies con las regiones vecinas; el CSNE comparte 151 de sus 161 especies con Brasil austral (Índice de Simpson $=0,94$ ), que es florísticamente más rico. El CSNO comparte 159 de sus 182 especies con Bolivia (Índice de Simpson $=0,87$ ) pero solo 68 especies con el CSNE (Índice de Simpson $=0,42$ ). Argentina tiene un fuerte intercambio florístico con todos los países vecinos de Sudamérica y forma juntamente con Uruguay una unidad florística como prueba el valor más alto del Índice de Simpson, mientras Chile presenta escaso vínculo florístico con el resto de Sudamérica, con excepción de Argentina (Tabla 5).

\section{Endemismos}

Chile continental tiene un endemismo $(11,2 \%)$ más alto que Argentina (3,5\%). Los endemismos continentales, tanto chilenos como argentinos, están restringidos a 8 géneros (Anexo 1). El CTS contiene el $77 \%$ de los endemismos continentales (44 especies sobre un total de 57 endémicas) (Tabla 3), que son compartidos o exclusivos del sur de Chile, con la excepción de Hymenophyllum quetrihuense, que es endémico de Neuquén y Río 
TABLA 4

Número de especies por provincia, especies compartidas e índice de Jaccard (comunes/[Prov. Argentina + Prov. Chile - compartidas]) para todas las combinaciones de provincias adyacentes de Argentina y Chile, ordenado de norte a sur

Species number per province, number of shared species and Jaccard index (common/[Prov. Argentina + Prov. Chile - shared]) for all combinations of adjacent provinces of Argentina and Chile, sorted from north to south

\begin{tabular}{lccccc}
\hline Provincia de Chile & $\begin{array}{c}\text { Número de } \\
\text { especies }\end{array}$ & $\begin{array}{c}\text { Provincia de } \\
\text { Argentina }\end{array}$ & $\begin{array}{c}\text { Número de } \\
\text { especies }\end{array}$ & $\begin{array}{c}\text { Número de especies } \\
\text { compartidas }\end{array}$ & $\begin{array}{c}\text { Índice de } \\
\text { Jaccard }\end{array}$ \\
\hline Antofagasta & 12 & Jujuy & 138 & 7 & 0,05 \\
Antofagasta & 12 & Salta & 148 & 8 & 0,05 \\
Atacama & 6 & Catamarca & 70 & 3 & 0,04 \\
Atacama & 6 & La Rioja & 31 & 4 & 0,12 \\
Coquimbo & 29 & San Juan & 23 & 5 & 0,22 \\
Valparaíso & 31 & Mendoza & 30 & 11 & 0,25 \\
Santiago & 25 & Mendoza & 30 & 11 & 0,24 \\
Libertador & 21 & Mendoza & 30 & 10 & 0,20 \\
Maule & 31 & Mendoza & 30 & 10 & 0,48 \\
Biobío & 56 & Neuquen & 55 & 36 & 0,49 \\
Araucanía & 58 & Neuquen & 55 & 37 & 0,59 \\
Los Lagos & 77 & Neuquen & 55 & 59 & 0,56 \\
Los Lagos & 77 & Río Negro & 62 & 50 & 0,35 \\
Los Lagos & 77 & Chubut & 32 & 28 & 0,48 \\
Aisén & 54 & Chubut & 32 & 28 & 0,43 \\
Aisén & 54 & Santa Cruz & 33 & 26 & 0,55 \\
Magallanes & 45 & Santa Cruz & 34 & 34 & 0,61 \\
Magallanes & 45 & Tierra del Fuego & 34 & 30 & \\
\hline
\end{tabular}

Negro en Argentina. En el CSNO se hallan 10 endemismos $(17,5 \%)$. Las tres especies endémicas restantes se encuentran fuera de los centros de diversidad: Isoëtes ekmanii crece en la Isla Martín García en el estuario del Plata, que está conectado por las selvas en galería a lo largo de los ríos de la Mesopotamia con el CSNE; y Cheilanthes sarmientoi en Argentina y Pellaea myrtillifolia en Chile, que se hallan en la Diagonal Árida.

\section{DISCUSIÓN}

Las floras pteridofíticas de Argentina y Chile son relativamente pobres en comparación con las floras de Perú, Bolivia y Brasil, que tienen entre tres y cuatro veces más especies (Foster 1958, Tryon \& Stolze 1989a, b, 1991, 1992, 1993, 1994, Palacios-Ríos \& Mehltreter 1998, Smith et al. 1999). En Chile las Pteridófitas nativas son el 2,3\% de las 5.100 especies de plantas vasculares (Marticorena 1990) y en Argentina forman el 3,6 $\%$ de las 9.690 especies de la flora vascular (Zuloaga et al. 1999).

Las Pteridófitas poseen en general una distribución más amplia que las Angiospermas (Tryon 1986, Stuessy et al. 1990), siendo el porcentaje de endemismos relativamente menor, tanto en conti- nentes como en islas. En las especies de Angiospermas el endemismo es entre cinco y seis veces mayor $(19,7 \%$ Argentina $50 \%$ Chile $)$ (Zuloaga et al. 1999 y Marticorena 1990, respectivamente) que en pteridófitas.

Sin embargo, como resultado de este trabajo encontramos que las Pteridófitas del Cono Sur sudamericano presentan tres centros de diversidad bien definidos, que concentran el $93 \%$ de las especies y el $95 \%$ de los endemismos, aunque difieren en composición florística entre ellos. ¿Por qué se concentran las especies solamente en tres centros de diversidad? ¿Por qué tienen los tres centros pteridofloras tan diferentes?

Los tres centros de diversidad coinciden con zonas montañosas con heterogeneidad de hábitats, en bosques y selvas con alta precipitación. Además existen algunos taxa (12\%) con distribuciones disyuntas en los tres centros, como por ejemplo las Hymenophyllaceae (HymenophyllumTrichomanes) y los géneros Blechnum (Blechnaceae), Elaphoglossum (Lomariopsidaceae), Hypolepis (Dennstaedtiaceae), Lycopodium y Huperzia (Lycopodiaceae), Megalastrum y Polystichum (Dryopteridaceae), que nos indican que la pteridoflora del Cono Sur fue históricamente más extensa y continua, y que sus límites han retrocedido a causa de los impactantes cam- 


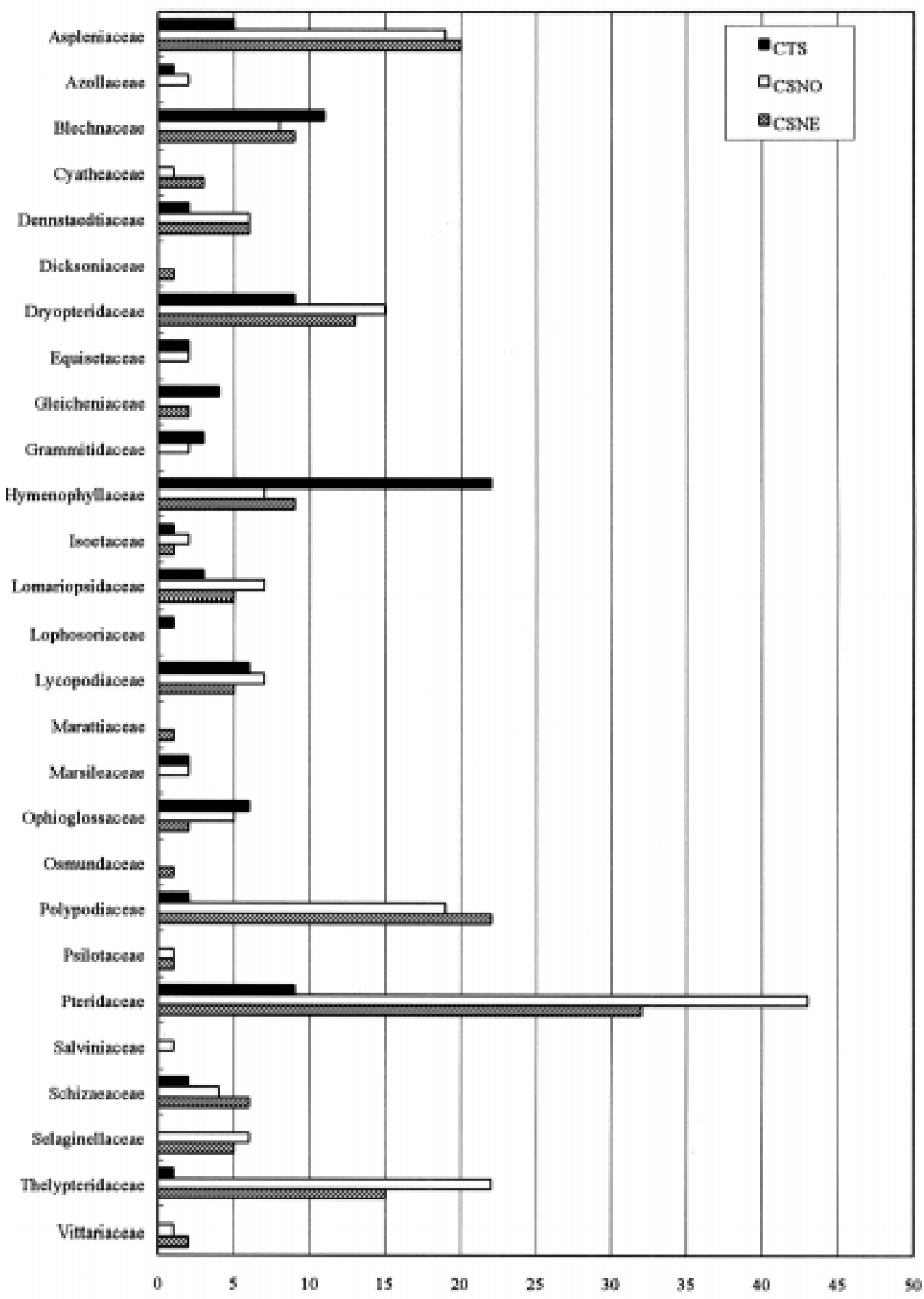

Fig. 4: Comparación florística entre los tres centros de diversidad pteridofítica: Centro Subtropical del NE (CSNE) de Argentina, Centro Subtropical del NO (CSNO) de Argentina, y Centro Templado del S (CTS) de Chile y Argentina.

Floristic comparison of the three centers of pteridophyte diversity: Subtropical Center of Northeastern Argentina (CSNE), Subtropical Center of Northwestern Argentina (CSNO) and Temperate Center of Southern Chile and Argentina (CTS). 
Relación de la flora pteridofítica argentino-chilena con países vecinos, expresado por el número de especies en común (a la derecha y arriba) y el Índice de Simpson (a la izquierda y abajo)

Floristic relationships of Argentinean-Chilean Pteridophytes with neighboring countries, expressed by the number of common species (on the right side and above) and Simpson's index (on the left side and below)

\begin{tabular}{lccccccc}
\hline & $\begin{array}{c}\text { Número de } \\
\text { especies }\end{array}$ & Argentina & Chile & Bolivia & Brasil & Uruguay & Paraguay \\
\hline Argentina & 346 & $*$ & 89 & 224 & 215 & 79 & 149 \\
Chile & 116 & 0,77 & $*$ & 43 & 26 & 13 & 14 \\
Bolivia & - & 0,65 & 0,37 & $*$ & - & 63 & - \\
Brasil & - & 0,62 & 0,22 & - & $*$ & 81 & - \\
Uruguay & - & 0,91 & 0,15 & 0,72 & 0,93 & $*$ & 66 \\
Paraguay & - & 0,43 & 0,12 & - & - & 0,76 & $*$ \\
\hline
\end{tabular}

- Datos incompletos

bios climáticos y geomorfológicos que sucedieron durante la segunda parte del Terciario, como el solevantamiento de los Andes, transgresiones oceánicas, ciclos de aridez y disminución de la temperatura (Hinojosa \& Villagrán 1997). Los actuales patrones de distribución están estrechamente ligados a las glaciaciones del Pleistoceno que afectaron cerca de dos tercios de los bosques de Sudamérica austral, produciendo así desplazamientos, compresión, y extinciones de floras (Villagrán \& Hinojosa 1997). El ejemplo más famoso es Thyrsopteris, paleoendemismo de las islas Juan Fernández, que se encuentra como fósil en yacimientos continentales del Cretácico (Tryon 1986, Moran 1993). Durante el Cretácico superior hasta el Eoceno se desarrollaron extensos bosques entre $\operatorname{los} 60-80^{\circ}$ de latitud sur (Stuessy \& Taylor 1995). Numerosas familias de Pteridófitas formaron parte de ellos, habiéndose encontrado fósiles de esos períodos y anteriores, de Cyatheaceae, Gleicheniacae, Dennstaedtiaceae, Osmundaceae en Patagonia (Archangelsky \& de la Sota 1963, Villar de Seoane 1996, 1999) y Cyatheaceae, Osmundaceae, y Marattiaceae en la Antártida (Gee 1989, Delevoryas et al. 1992, Phipps et al. 1998, Césari et al. 1999²). Muchos de estos taxa se extinguieron debido a los cambios paleoclimáticos. Lo mismo pudiera haber pasado durante el Pleistoceno, cuando el extremo austral de Sudamérica al sur de los $43^{\circ}$ estuvo cubierto por

${ }^{2}$ CÉSARI S, M REMESAL \& C PARICA (1999)

Ferns: A significant component of the Cretaceous Flora from Livingston Island, Antarctica. VII International Symposium on Mesozoic Terrestrial Ecosystem. Buenos Aires, Abstracts. glaciares (Vuilleumier 1971, Hinojosa \& Villagrán 1997) extinguiéndose floras subtropicales. Como consecuencia de estas extinciones se empobreció la flora de los bosques templados lluviosos en tal forma, que el $86 \%$ de los géneros de plantas con semilla están actualmente representados por 1-3 taxa, siendo en general el $90 \%$ endemismos chileno-argentinos (Villagrán \& Hinojosa 1997). En estos bosques pocos géneros de plantas vasculares están representados con más especies, entre ellas Blechnum (10) e Hymenophyllum (21).

En el caso de las Hymenophyllaceae, el género Trichomanes se encuentra en ambos centros subtropicales (CSNO y CSNE), con la excepción de Trichomanes exsectum que habita las regiones de Valdivia y Chiloé, las cuales están ubicadas dentro de la llamada "selva valdiviana", sin duda el área más cálida y lluviosa dentro de los bosques andino-patagónicos (Hauman 1916). Al contrario de Trichomanes, el género Hymenophyllum tiene una distribución con casi la totalidad de las especies en el centro templado sur (CTS), siendo muchas de ellas endémicas.

La distribución del género Blechnum es disyunta en los tres centros de diversidad, y con algunas especies o complejos de especies en las zonas intermedias como Sierras Australes de Buenos Aires, Sierras de Córdoba y Chile central. Algunos ejemplos son $B$. chilense-B. cordatum de Perú (Tryon \& Stolze, 1993) y B. magellanicum- $B$. cycadifolium-B. tabulare (de la Sota, 1977b). Además varias de sus especies son endémicas (Anexo 1). Este tipo de distribución contribuiría a las evidencias de la continuidad de los bosques subtropicales en el pasado (Troncoso et al. 1980).

Los tres centros de diversidad tienen pocas especies en común (Tabla 3 ), sea por consecuencia de las ya discutidas extinciones en el 
Pleistoceno, sea por un diferente origen de sus floras, o sea por su fuerte aislamiento. Las diferencias más pronunciadas existen entre el CTS y los dos centros subtropicales. El CTS tiene una flora dominada por Hymenophyllaceae y Blechnaceae, familias con un alto grado de endemismo, que evidentemente diversificaron allí. Además, el mayor endemismo indica un fuerte aislamiento entre el CTS y los dos centros subtropicales debido a las barreras de la Diagonal Árida y Los Andes. Estas barreras restringieron la dispersión en ambas direcciones, por lo que el 77 $\%$ de los endemismos continentales argentinochilenos se concentran en el CTS. Muchas especies de familias dominantes en los centros subtropicales como Aspleniaceae, Polypodiaceae, y Thelypteridaceae, consideradas modernas dentro de los leptosporangiados, no han extendido su área de distribución hasta el CTS.

El CTS presenta relaciones florísticas históricas con las zonas austral-antárticas, con predominio Pacífico, y Sudafricano, como en el caso de Blechnum australe L. subsp. auriculatum y el caso particular de B. magellanicum vs. B. tabulare (de la Sota 1977a, 1977b, de la Sota \& Cassá de Pazos 1983). Además, comparte todavía el $30 \%$ de su flora pteridofítica con Bolivia, aunque se trata principalmente de especies de amplia distribución dentro del continente sudamericano.

Dentro del CTS mismo encontramos el intercambio florístico más fuerte entre ambas vertientes de Los Andes (Tabla 4), debido a la menor elevación de Los Andes australes. Además existen en el CTS numerosos valles diferentemente orientados que conectan las floras entre ambas vertientes de la cordillera, por ejemplo el paso andino del Lago Puelo a $200 \mathrm{~m}$ de altitud que es una vía de penetración de taxa valdivianos montanos como Hymenoglossum cruentum, Hymenophyllum caudiculatum var. productum e H. fuciforme hacia Los Andes orientales (Vidoz et al. 1999).

Los dos centros subtropicales están fuertemente vinculados con las floras tropicales de Bolivia y Brasil, de cuales no les separa ninguna barrera efectiva. El CSNO tiene alta similitud con Bolivia, y contiene apenas $17,5 \%$ de los endemismos, un porcentaje bajo que denota la amplia distribución de los helechos a lo largo de las Yungas, que comparte con el sur de Bolivia. El CSNE tiene alta similitud con Brasil y ninguna especie endémica como consecuencia de la continuidad de las selvas del sureste de Brasil y la selva de Misiones en Argentina.

Concluimos que la flora pteridofítica del Cono Sur se concentra en tres centros de diversidad. Las pteridofloras del CSNO y CSNE están vinculadas fuertemente con las floras de Bolivia y
Brasil, respectivamente, por lo que tienen un endemismo muy bajo. Al contrario, el CTS es el más aislado y con mayor endemismo, pero su diversidad es menor como consecuencia del aislamiento y de extinciones durante el Pleistoceno.

\section{AGRADECIMIENTOS}

Los autores agradecen a los colegas de sus Instituciones por los consejos y apoyo en la discusión de este trabajo. El segundo autor manifiesta puntualmente su agradecimiento a la Sra. Christel Necker, Departamento de Biología V, Universidad de Ulm, por su apoyo en la búsqueda bibliográfica. También agradecen a los revisores anónimos por los comentarios que mejoraron la discusión sustancialmente.

\section{LITERATURA CITADA}

ARCHANGELSKY S \& ER DE LA SOTA (1963) Osmunda herbstii, nueva petrificación triásica de "E1 Tranquilo". Ameghiniana 3: 135-140.

ARGENTINA-INSTITUTO GEOGRÁFICO MILITAR (1998) Atlas de la República Argentina. Séptma edición. Instituto Geográfico Militar, Buenos Aires, Argentina. $95 \mathrm{pp}$.

BARRINGTON DS (1993) Ecological and historical factors in fern biogeography. Journal of Biogeography 20: 275-280.

BROWN JH \& MV LOMOLINO (1998) Biogeography. Second edition. Sinauer Associates, Sunderland, Massachussetts. xii $+692 \mathrm{pp}$.

CABRERA AL \& A WILLINK (1973) Biogeografía de América Latina. Programa Regional de Desarrollo Científico y Tecnológico, Departamento de Asuntos Científicos, Secretaría General de la Organización de los Estados Americanos, Washington, District of Columbia. $120 \mathrm{pp}$.

CHILE-INSTITUTO GEOGRÁFICO MILITAR (1985) Atlas geográfico de Chile para la educación. Primera edición. Instituto Geográfico Militar, Santiago, Chile. $140 \mathrm{pp}$.

DE LA SOTA ER (1972) Sinopsis de las Pteridófitas del noroeste de Argentina, I. Darwiniana 17: 11-103.

DE LA SOTA ER (1973a) La distribución geográfica de las Pteridófitas en el Cono Sur de América meridional. Boletín de la Sociedad Argentina de Botánica 15: 23-34.

DE LA SOTA ER (1973b) Sinopsis de las Pteridófitas del noroeste de Argentina, II. Darwiniana 18: 173-263.

DE LA SOTA ER (1976) Sinopsis de las Pteridófitas del noroeste de Argentina, III. Darwiniana 20: 225-232.

DE LA SOTA ER (1977a) Sinopsis de las Pteridófitas del noroeste de Argentina, IV. Darwiniana 21: 120-138.

DE LA SOTA ER (1977b) Pteridophyta. En: Cabrera AL (ed) Flora de la Provincia de Jujuy, Republica Argentina: 1-275. Colección Científica, Instituto Nacional de Tecnología Agropecuaria 13, Buenos Aires, Argentina. 
DE LA SOTA ER \& L CASSÁ DE PAZOS (1983) Contribución al conocimiento biosistemático de las especies austrosudamericanas del género Blechnum L. (Blechnaceae, Pteridophyta). Lilloa 36: 77-83.

DE LA SOTA ER, MM PONCE, MA MORBELLI \& L CASSÁ DE PAZOS (1998) Pteridophyta. En: Correa MN (ed) Flora patagónica: 282-369. Colección Científica, Instituto Nacional de Tecnologia Agropecuaria 8, Buenos Aires, Argentina.

DELEVORYAS T, TN TAYLOR \& EL TAYLOR (1992) A marattialean fern from the Triassic of Antarctica. Review of Palaeobotany and Palynology 74: 101-107.

DUEK JJ \& R RODRÍGUEZ (1972) Lista preliminar de las especies de Pteridophyta en Chile continental e insular. Boletín de la Sociedad de Biología de Concepción (Chile) 45: 129-174.

FOSTER RC (1958) A catalogue of ferns and flowering plants of Bolivia. Contributions of the Gray Herbarium 184: 1-223.

GEE CT (1989) Revision of the Late Jurassic/Early Cretaceous flora from Hope Bay, Antarctica. Palaeontographica, Abteil B, Paläophytology 213: 149-214.

GODOY R \& H FIGUEROA (1989) Composition and distribution of the pteridophyte flora of continental and insular Chile. Nova Hedwigia 48: 437-453.

HASSLER E (1928) Pteridophytorum Paraguariensium. Trabajos del Instituto de Botánica y Farmacología (Argentina) 45: 1-102.

HAUMAN L (1916) La forêt valdivienne et ses limites. Instituto de Botánica y Farmacología (Argentina) 34: $1-91$.

HICKEN CM (1919) La migración de los helechos en la flora de Tucumán. Primera Reunión Nacional de la Sociedad Argentina de Ciencias Naturales, Tucumán 1916: 187-209. Sociedad Argentina de Ciencias Naturales, Imprenta CONI, Buenos Aires Argentina.

HINOJOSA LF \& C VILLAGRÁN (1997) Historia de los bosques del sur de Sudamérica I: antecedentes paleobotánicos, geológicos y climáticos del Terciario del cono sur de América. Revista Chilena de Historia Natural 70: 225-239.

KATO M (1993) Biogeography of ferns: dispersal and vicariance. Journal of Biogeography 20: 265-274.

KRAMER KU \& PS GREEN (eds.) (1990) Pteridophytes and gymnosperms. En: Kubitzki K (ed) The families and genera of vascular plants, Volume I: 11-277, Springer Verlag, Berlin, Germany.

KRAMER KU, JJ SCHNELLER \& E WOLLENWEBER (1995) Farne und Farnverwandte, Erste Auflage. Thieme Verlag, Stuttgart, Germany. 198 pp.

LOOSER G (1932) Ensayo sobre la distribución geográfica de los helechos chilenos. Revista Chilena de Historia y Geografía 69: 162-198.

LOOSER G (1948) The ferns of Southern Chile. American Fern Journal 38(2): 33-44.

LOOSER G (1955) Los helechos de Chile central. Moliniana 1: 5-95.

LOOSER G (1961) Los Pteridofitos o helechos de Chile, I. Revista Universitaria (Chile) 46: 213-262.

LOOSER G (1962) Los Pteridofitos o helechos de Chile, II. Revista Universitaria (Chile) 47:17-31.

LOOSER G (1966) Los Pteridofitos o helechos de Chile, III. Revista Universitaria (Chile) 50-51: 75-93.
LOOSER G (1968) Los Pteridofitos o helechos de Chile, IV. Revista Universitaria (Chile) 53: 27-39.

MARTICORENA C (1990) Contribución a la estadística de la flora vascular de Chile. Gayana Botánica (Chile) $47: 85-114$.

MORAN RC (1993) Robinson Crusoe's ferns. Fiddlehead Forum 20: 44-47.

PALACIOS-RIOS M \& K MEHLTRETER (1998) A database for the knowledge and conservation of the biodiversity of Latin American pteridophytes. American Journal of Botany (Supplement) 85: 97.

PHIPPS CJ, TN TAYLOR, EL TAYLOR, NR CÚNEO, LD BOUCHER \& X YAO (1998) Osmunda (Osmundaceae) from the Triassic of Antarctica: an example of evolutionary stasis. American Journal of Botany 85: 888-895.

PONCE MM (1994) Pteridófitos. En: Kiesling R (ed) Flora de San Juan, República Argentina, Volumen 1: 17-39. Vázquez Mazzini Editores, Buenos Aires, Argentina.

PONCE MM (1995) Las especies austrobrasileñas de Thelypteris subgénero Amauropelta (Thelypteridaceae, Pteridofita). Darwiniana 33: 257 283.

PONCE MM (1996) Pteridophyta. En: Zuloaga FO \& O Morrone (eds) Catálogo de las plantas vasculares de la República Argentina, Parte I. Monographs of Systematic Botany, Missouri Botanical Garden 60: 179.

RODRÍGUEZ RR (1989) Comentarios fitogeográficos y taxonómicos de Pteridophyta chilenos. Gayana Botánica 46: 199-208.

RODRÍGUEZ RR (1995a) Pteridophyta. En: Simonetti JA, MTK Arroyo, AE Spotorno \& E Lozada (eds.) Diversidad biológica en Chile: 58-65. Comisión Nacional de Ciencia y Tecnología, Santiago, Chile.

RODRÍGUEZ RR (1995b) Pteridophyta. En: Marticorena C \& RR Rodríguez (eds) Flora de Chile, Volumen I: 119-337. Ediciones de la Universidad de Concepción, Concepción, Chile.

SMITH AR (1993) Phytogeographic principles and their use in understanding fern relationships. Journal of Biogeography 20: 255-264.

SMITH AR, M KESSLER \& J GONZÁLES (1999) New records of pteridophytes from Bolivia. American Fern Journal 89: 244-266.

SQUEO FA, L CAVIERES, G ARANCIO, JE NOVOA, O MATTHEI, C MARTICORENA, R RODRÍGUEZ, MTK ARROYO \& M MUÑOZ (1998) Biodiversidad vegetal de Antofagasta. Revista Chilena de Historia Natural 71: 571-591.

STUESSY TF, DJ CRAWFORD \& C MARTICORENA (1990) Patterns of phylogeny in the endemic vascular flora of the Juan Fernandez Islands, Chile. Systematic Botany 15: 338-346.

STUESSY TF \& C TAYLOR (1995) Evolución de la flora chilena. En: Marticorena C \& RR Rodríguez (eds) Flora de Chile, Volumen I: 85-118. Ediciones de la Universidad de Concepción, Concepción, Chile.

TRONCOSO A, C VILLAGRÁN \& M MUÑOZ (1980) Una nueva hipótesis acerca del origen y edad del bosque de Fray Jorge (Coquimbo, Chile). Boletín del Museo Nacional de Historia Natural (Chile) 37: 117 152 . 
TRYON RM (1986) The biogeography of species, with special reference to ferns. Botanical Review 52: 117156.

TRYON RM \& RG STOLZE (1989a) Pteridophyta of Peru, Part I, 1. Ophioglossaceae - 12. Cyatheaceae. Fieldiana Botany, New Series 20: 1-145.

TRYON RM \& RG STOLZE (1989b) Pteridophyta of Peru, Part II, 13. Pteridaceae - 15. Dennstaedtiaceae. Fieldiana Botany, New Series 22: 1-128.

TRYON RM \& RG STOLZE (1991) Pteridophyta of Peru, Part IV, 17. Dryopteridaceae. Fieldiana Botany, New Series 27: 1-176.

TRYON RM \& RG STOLZE (1992) Pteridophyta of Peru, Part III, 16. Thelypteridaceae. Fieldiana Botany, New Series 29: 1-80.

TRYON RM \& RG STOLZE (1993). Pteridophyta of Peru, Part V, 18. Aspleniaceae - 21. Polypodiaceae. Fieldiana Botany, New Series 32: 1-190.

TRYON RM \& RG STOLZE (1994) Pteridophyta of Peru, Part VI, 22. Marsileaceae - 28. Isoëtaceae. Fieldiana Botany, New Series 34: 1-123.

VIDOZ FF, LA CASSA DE PAZOS \& ER DE LA SOTA (1999) Paso Puelo, Chubut, Argentina: vía de ingreso de helechos valdivianos. Parodiana 11: 43-48.

VILLAGRÁN C \& JJ ARMESTO (1980) Relaciones florísticas entre las comunidades relictuales del Norte Chico y la zona central con el bosque del sur de Chile. Boletín del Museo Nacional de Historia Natural (Chile) 37: 87-101.
VILLAGRÁN C \& LF HINOJOSA (1997) Historia de los bosques de Sudamérica. II: Análisis fitogeográfico. Revista Chilena de Historia Natural 70: 241-267.

VILLAGRÁN C, P MORENO \& R VILLA (1996) Antecedentes pelinológicos acerca de la historia Cuaternaria de los bosques chilenos. En: Armesto JJ, C Villagrán \& MTK Arroyo (eds) Ecología de los bosques nativos de Chile: 51-69. Editorial Universitaria, Santiago, Chile.

VILLAR DE SEOANE L (1996) Estudio morfológico de helechos fértiles hallados en la Formación Baqueró (Cretácico Inferior), Provincia de Santa Cruz, Argentina. Revista Española de Paleontología 11: 83-90.

VILLAR DE SEOANE L (1999) Estudio comparado de Cyathea cyathifolia combinatio nova del Cretácico inferior de Patagonia, Argentina. Revista Española de Paleontología 14: 157-163.

VUILLEUMIER BS (1971) Pleistocene changes in the fauna and flora of South America. Science 173: 771780.

ZAR JH (1999) Biostatistical analysis. Fourth edition. Prentice-Hall, Inc., Englewood Cliffs, New Jersey. $718 \mathrm{pp}$.

ZULOAGA FO, O MORRONE \& D RODRÍGUEZ (1999) Análisis de la biodiversidad en plantas vasculares de la Argentina. Kurtziana 27: 17-167. 
ANEXO 1

\section{Lista de taxa de Pteridófitas consideradas en este estudio}

List of Pteridophyte taxa here considered

Géneros con especies nativas en Argentina pero ausentes en Chile continental

Genera of native species of Argentina absent in continental Chile

Acrostichum, Adiantopsis, Alsophila, Anogramma, , Athyrium, Bolbitis, Campyloneurum, Ceratopteris, Ctenitis, Cyathea, Cyclodium, Cheiroglossa, Dicksonia, Dicranopteris, Didymochlaena, Diplazium, Doryopteris, Dryopteris, Hemionitis, Lastreopsis, Lindsaea, Lellingeria, Lomagramma ${ }^{3}$, Lycopodiella, Lygodium, Marattia, Melpomene, Microgramma, Microlepia ${ }^{3}$, Niphidium, Osmunda, Pecluma, Phlebodium, Phyllitis, Pityrogramma, Polytaenium, Psilotum, Pteridium, Regnellidium, Saccoloma ${ }^{3}$, Selaginella, Tectaria, Trachypteris, Trichipteris y Vittaria

Género ausente en Argentina, pero presente en Chile Genus absent in Argentina, present in Chile

\section{Histiopteris}

Pteridófitas endémicas fuera de los Centros de diversidad, (3 spp.)

Endemic pteridophyte outside of diversity centers

Cheilanthes sarmientoi Ponce

Isoëtes ekmanii U. Weber

Pellaea myrtillifolia Mett. ex Kuhn

Pteridófitas endémicas del CSNO (Argentina), (10 spp. + 2 taxa subespecíficos $=12$ taxa)

Endemic pteridophyte from CSNO

Asplenium achalense Hieron.

Asplenium lilloanum de la Sota

Blechnum mochaenum G. Kunkel var. squamipes (Hieron.) de la Sota

Campyloneurum tucumanense (Hieron.) Ching

Hymenophyllum capurroi de la Sota

Hymenophyllum tunbrigense (L.) Sm. var. cordobense Hieron.

Isoëtes alcalophila Halloy

Isoëtes escondidensis Halloy

Lellingeria tungurahuae (Rosenst.)A.R.Smith \& R.C.Moran

Pecluma oranense (de la Sota) de la Sota

Pteris famatiensis de la Sota

Thelypteris jujuyensis de la Sota
Pteridófitas endémicas del CTS (Argentina y Chile), (32 spp. +12 taxa subespecíficos $=44$ taxa)

Endemic pteridophyte from CTS

Adiantum chilense Kaulf. var. chilense

Adiantum chilense Kaulf. var. scabrum (Kaulf.) Hicken

Adiantum chilense Kaulf. var. subsulphureum (Hook.)

Giudice

Asplenium dareoides Desv.

Blechnum arcuatum J. Rémy

Blechnum chilense (Kaulf.) Mett.

Blechnum hastatum Kaulf.

Blechnum magellanicum (Desv.) Mett.

Blechnum microphyllum (Goldm.) C.V. Morton

Botrychium australe R. Br. subsp. negeri (H. Christ) R.T.

Clausen

Botrychium dusenii (H. Christ) Alston

Cheilanthes glauca (Cav.) Mett.

Cheilanthes hypoleuca (Kunze) Mett.

Cryptogramma fumariifolia (Phil. ex Baker) H. Christ

Elaphoglossum fonckii (Phil.) T. Moore

Elaphoglossum porteri Hicken

Gleichenia cryptocarpa Hook.

Gleichenia quadripartita (Poir.) T. Moore

Hymenophyllum darwinii Hook.

Hymenophyllum dentatum Cav.

Hymenophyllum falklandicum Baker

Hymenophyllum krauseanum Phil.

Hymenophyllum nahuelhuapiense Diem \& J.S. Licht.

Hymenophyllum pectinatum Cav.

Hymenophyllum plicatum Kaulf.

Hymenophyllum quetrihuense Diem \& J.S.Licht.

Hymenophyllum secundum Hook. \& Grev.

Hymenophyllum tortuosum Hook. \& Grev.

Hymenophyllum umbratile Diem \& J.S. Licht.

Isoëtes hieronymi $\mathrm{U}$. Weber

Isoëtes savatieri Franch.

Lycopodium alboffii Rolleri

Lycopodium confertum Willd.

Lycopodium gayanum J. Rémy

Lycopodium paniculatum Desv. ex Poir.

Megalastrum spectabile var. spectabile

Ophioglossum crotalophoroides Walter subsp. robustum

R.T.Clausen

Pleurosorus papaverifolius (Kunze) Fée

Polypodium feuillei Bertero var. feuillei

Polystichum andinum Phil.

Polystichum chilense (H. Christ) Diels var. chilense

Polystichum chilense var. dusenii

Polystichum multifidum (H. Christ) Diels var. multifidum

Polystichum multifidum (H. Christ) Diels var. pearcei (Phil.) R. Rodr.

\footnotetext{
${ }^{3}$ Lomogramma, Microlepia y Saccoloma fueron citadas en Hassler (1928) para Misiones, estos especímenes de herbario no se han visto
} 
ANEXO 1 (continuación)

\section{Lista de taxa de Pteridófitas consideradas en este estudio}

List of Pteridophyte taxa here considered

Polystichum plicatum (Poepp. ex Kunze) Hicken

Pteris semiadnata Phil.

Serpyllopsis caespitosa (Gaudich.) C.Chr. var. caespitosa

Pteridófitas endémicas de Argentina (12 spp. + 4 taxa subespecíficos $=16$ taxa)

Endemic pteridophyte of Argentina

Adiantum thalictroides Willd. ex Schltdl. f. bottini Giudice \& Nieto

Asplenium achalense Hieron.

Asplenium lilloanum de la Sota

Blechnum mochaenum G. Kunkel var. achalense (Hieron.) de la Sota

Blechnum mochaenum G. Kunkel var. squamipes (Hieron.) de la Sota

Campyloneurum tucumanense (Hieron.) Ching

Cheilanthes sarmientoi Ponce

Hymenophyllum capurroi de la Sota

Hymenophyllum quetrihuense Diem \& J.S.Licht.

Hymenophyllum tunbrigense (L.) Sm. var. cordobense Hieron.

Isoëtes alcalophila Halloy

Isoëtes ekmanii U.Weber

Isoëtes escondidensis Halloy

Pecluma oranense (de la Sota) de la Sota

Pteris famatiensis de la Sota

Thelypteris jujuyensis de la Sota

Pteridófitas endémicas de Chile continental (13 spp. + 4 taxa subespecíficos $=17$ taxa)

Endemic pteridophyte of continental Chile

Adiantum excisum Kunze

Adiantum gertrudis Espinosa

Adiantum pearcei Phil.

Adiantum sulphureum Kaulf.

Blechnum asperum (Klotzsch) Sturm

Blechnum corralense Espinosa

Elaphoglossum fonckii (Phil.) T. Moore

Elaphoglossum porteri Hicken

Gleichenia litoralis (Phil.) C.Chr.

Gleichenia squamulosa (Desv.) T. Moore var. gunckeliana (Looser) Duek

Hymenophyllum dicranotrichum (C.Presl) Hook. ex. Sadeb. Megalastrum spectabile (Kaulf.) A.R. Sm. \& R.C. Moran var. philippianum (C. Chr.) A.R. Sm. \& R.C. Moran

Ophioglossum nudicaule L. f. var. robustum J.S. Licht.

Pellaea myrtillifolia Mett. ex Kuhn

Polypodium espinosae Weath.

Polypodium feullei Bertero var. ibañezii Looser

Polystichum subintegerrimum (Hook. \& Arn.) R.A.Rodr.

Pteridófitas endémicas de Chile continental e insular (4 spp. +4 taxa subespecíficos $=8$ taxa)

Endemic pteridophyte of continental and insular Chile
Asplenium obtusatum G. Foster var. sphenoides (Kunze)

C. Chr. ex Skottsb.

Gleichenia squamulosa (Desv.) T. Moore var. squamulosa Hymenoglossum cruentum (Cav.) C.Presl

Hymenophyllum caudiculatum Mart. var. productum (C. Presl) C. Chr.

Hymenophyllum cuneatum Kunze var. cuneatum

Hymenophyllum fuciforme $\mathrm{Sw}$.

Trichomanes exsectum Kunze

Pteris chilensis Desv.

Pteridófitas endémicas del archipiélago Juan Fernández (18 spp. +7 taxa subespecíficos $=25$ taxa $)$

Endemic pteridophyte of Juan Fernández archipielago

Arthropteris altescandens (Colla) J.Sm.

Asplenium macrosorum Bertero ex Colla

Asplenium stellatum Colla

Blechnum cycadifolium (Colla) Sturm

Blechnum longicauda C.Chr.

Blechnum mochaenum G. Kunkel var. fernandezianum (Looser) de la Sota

Blechnum schottii (Colla) C.Chr.

Dicksonia berteriana (Colla) Hook.

Dicksonia externa C.Chr. \& Skottsb.

Gleichenia lepidota R.A.Rodr.

Hymenophyllum cuneatum Kunze var. rariforme C. Chr. \& Skottsb.

Hymenophyllum rugosum C.Chr. \& Skottsb.

Megalastrum inaequalifolium (Colla) A.R. Sm \& R.C. Moran var. glabrior (C. Chr. \& Skottsb.) R.A. Rodr.

Megalastrum inaequalifolium (Colla) A.R. Sm \& R.C. Moran var. inaequalifolium

Argyrochosma chilensis (J.Rémy) Windham

Ophioglossum fernandezianum C.Chr.

Polypodium intermedium Colla subsp. intermedium

Polypodium intermedium Colla subsp. masafueranum C.

Chr. \& Skottsb.

Polystichum tetragonum Fée

Pteris berteroana J.Agardh

Rumohra berteroana (Colla) R.A.Rodr.

Serpyllopsis caespitosa (Gaudich.) C.Chr. var. fernandeziana C.Chr. \& Skottsb.

Thyrsopteris elegans Kunze

Trichomanes ingae C.Chr.

Trichomanes philippianum Sturm

Pteridófitas endémicas de Isla de Pascua (4 spp + 1 taxa subespecíficos $=5$ taxa)

Endemic pteridophyte of Easter Island

Asplenium polyodon G. Foster var. squamulosum (C.Chr.)

R.A. Rodr.

Diplazium fuenzalidae Espinosa

Doodia paschalis C.Chr.

Elaphoglossum skottsbergii Krajina

Polystichum fuentesii Espinosa 